\title{
Integration of Fluorescent Functionality into Pressure Amplifying Metal-Organic Frameworks
}

\author{
Francesco Walenszus, Jack D. Evans ${ }^{\dagger}$, Volodymyr Bon, Friedrich Schwotzer, Irena \\ Senkovska, Stefan Kaskel* \\ Anorganische Chemie I, Fakultät Chemie und Lebensmittelchemie, Technische Universität Dresden, \\ Bergstrasse 66, 01062 Dresden, Germany
}

\begin{abstract}
The flexibility of soft porous crystals, i.e., their ability to respond to external stimuli with structural changes, is one of the most fascinating features of metal-organic frameworks (MOFs). In addition to breathing and swelling phenomena of flexible MOFs, negative gas adsorption (NGA) and pressure amplification (PA) is one of the more recent discoveries in this field, initially observed in the cubic DUT-49 framework. In recent years the structural contraction was monitored by physisorption, X-ray diffraction, NMR and EPR techniques, providing only limited information about the electronic structure of the ligand. In this work we designed a new ligand with a fluorescent core in the linker backbone and synthesized three new MOFs, isoreticular to DUT-49, denoted as DUT-140(M) (M - Cu, Co, $\mathrm{Zn}$ ) crystalizing in space group Fm $3 \mathrm{~m}$. DUT-140(Cu) can be desolvated and is highly porous with an accessible apparent surface area of $4870 \mathrm{~m}^{2} \mathrm{~g}^{-1}$ and a pore volume of $2.59 \mathrm{~cm}^{3} \mathrm{~g}^{-1}$. Furthermore, it shows flexibility and NGA upon adsorption of subcritical gases. DUT-140(Zn), synthesized using post-synthetic metal exchange, could only be studied with guests in the pores. In addition to the investigation of the adsorption behavior of DUT-140(Cu) spectroscopic and computational methods were used to study the light absorption properties.
\end{abstract}

\section{INTRODUCTION}

Over the last two decades, metal-organic frameworks (MOFs) have been established as a new class of highly porous coordination networks consisting of inorganic nodes (metal ions or metal-oxo clusters) and linker molecules. In this context, linkers are defined as polydentate organic ligands which interconnect the inorganic nodes. ${ }^{1-4}$ MOFs contain voids accessible for various probe molecules that can be used for many applications. ${ }^{5}$ Due to this modular design, there are almost no limits regarding structural nodes combination, resulting in a wide variety of frameworks with different topologies. In addition to excellent tunability of the material properties, MOFs often show immense surface areas ${ }^{6,7}$ with permanent porosity. A limited number of MOFs show also structural flexibility, reflected in a distinct structural response to external stimuli which can be considered among the most unique and fascinating properties compared to traditional porous solids. The phenomenon was first predicted in $1998^{8}$ as an attribute of the third generation of MOFs, which are also known as flexible MOFs or soft porous crystals. ${ }^{2}$ Dynamic behavior of soft porous crystals could be exploited in the last decades in a range of different applications like gas separation ${ }^{9-11}$, molecular sensing ${ }^{12-14}$, drug delivery ${ }^{15,16}$ and improved storage and release of gases $^{17}$ outperforming the rigid analogues. Different types of flexibility phenomena such as breathing, swelling, and subnetwork displacement have been described ${ }^{6,18}$, reflected in very specific adsorption characteristics and isotherm shapes. ${ }^{19}$

In 2016 a previously unknown phenomenon, later denoted as Negative Gas Adsorption (NGA), was discovered in the metal organic framework DUT-49, reflected by a spontaneous gas desorption and pressure amplification upon breathing. DUT-49 is build from tetratopic 9,9'([1,1'-biphenyl]-4,4'-diyl)-bis( $9 H$-carbazole-3,6dicarboxylate) ligand (BBCDC) and copper paddle wheel units. From a structural point of view, the material consists of cuboctahedral metal-organic polyhedra (MOP) formed by copper paddle wheels and 3,6-carbazole-dicarboxylates which are interconnected by 4,4'-biphenyl units forming close cubic packing. This linkage generates a hierarchical pore system of cuboctahedral (10 $\AA$ ), tetrahedral (17 $\AA)$ and octahedral (24 $\AA$ ) pores. During adsorption of subcritical gases at conditions close to the standard boiling point, the network shows a transition to a contracted pore phase (cp) while previously adsorbed gas is expelled, leading to a pressure amplification (PA) in the measurement cell. This transformation is, different to other materials, uniquely caused by a 
switch-like, out of plane buckling of the $\mathrm{sp}^{2}$ hybridzed biphenyl part of the BBCDC linker. ${ }^{20,21}$ Molecular simulations have highlighted the responsible for the contraction during methane adsorption $^{22}$ and in later work also the effect of crystallite size $^{23}$ and pore size in an isoreticular series $^{24}$ on the mechanical properties and the transition were investigated. Recent research on this material enlightens the switching behavior and the effect of defects using NMR techniques. ${ }^{25,26}$ Furthermore the impact of temperature and choice of adsorbate on the NGA step was examined more in detail. ${ }^{27,28}$ Today pressure amplification by NGA can approach higher pressures beyond $400 \mathrm{kPa}$ which may be valuable for pneumatic applications. ${ }^{29}$

The guest dependent flexibility of DUT-49 was extensively investigated by in situ X-ray diffraction/adsorption experiments, computational methods ${ }^{20,22-24}$ and in situ NMR experiments. ${ }^{25,26,30}$ However, none of these techniques addresses the changes in electronic states of the ligand molecule in detail. This aspect can be tackled by optical spectroscopic techniques and would give a deeper insight into changes in the electronic and molecular structure of the linker in response to the contraction trajectory. Further investigation of the linker in the closed pore phase allows a consideration of the question if the contraction of the network can be used to arrest a fragment of the linker in unnatural conformational states. This can be interesting for uncommon fluorescence properties but also for activation of reactive centers by increasing the accessibility for example.

In 2002, fluorescence was mentioned for the first time in connection with MOFs. ${ }^{31}$ Since then, different strategies were used to integrate fluorescent moieties into MOFs especially for sensing applications. ${ }^{32,33}$ For instance, it was found that the stiffening of luminophores in networks increases their fluorescence intensity because of decreased vibrational decay. Furthermore, the precise spatial fixation of fluorophores within a lattice can significantly influence the fluorescence properties and lead to interesting effects like J dimer emission ${ }^{34}$ or multi emission. ${ }^{35,36}$ Also, phenomena like charge and energy transfer, known from classical coordination chemistry, were observed. ${ }^{31,37}$ In addition to pure ligand-based fluorescence, MOFs containing $\mathrm{d}^{10}$ ions often exhibit metal-to-ligand (MLCT) and ligand-to-metal charge transfer (LMCT). ${ }^{31}$ Alternatively, fluorophores can be introduced into the pore system. which can lead to interesting emission properties. ${ }^{36}$ Another possibility is offered by lanthanide based MOFs in which highly absorbing linker molecules are used to populate the emissive states of the lanthanide and to increase its luminescence. ${ }^{31,38,39}$

In this work a fluorescent probe was introduced into DUT-49 framework. For this reason, the biphenyl moiety in the $\mathrm{H}_{4} \mathrm{BBCDC}$ ligand of DUT-49 was exchanged by fluorenone, due to the structural similarity and therefore, expected NGA behavior. To examine the possibility of NGA by using the 9,9'-(9-oxo-9H-fluorene-2,7-diyl)-bis(9Hcarbazole-3,6-dicarboxylic acid, $\mathrm{H}_{4} \mathrm{FBCDC}$ ) as linker a computational study on the mechanical properties of the ligand was conducted and compared with the $\mathrm{H}_{4} \mathrm{BBCDC}$ ligand. Subsequently, the fluorenone based ligand was synthesized and incorporated into DUT-140(Cu), a MOF isoreticular to DUT-49. The adsorption properties of DUT-140(Cu) were investigated and NGA behavior was observed upon adsorption of methane at $111 \mathrm{~K}$. To enable the fluorescence detection and to avoid paramagnetic fluorescence quenching, DUT-140(Zn) was synthesized by post synthetic metal exchange. The influence of the mechanical stress and linker deformation on fluorescence properties was further investigated in desolvation experiments.

\section{EXPERIMENTAL SECTION}

\section{Synthesis of microcrystalline MOF powders}

The microcrystalline powder of metal organic framework DUT-140(Cu) was synthesized by a solvothermal approach. In a $500 \mathrm{ml}$ round bottom flask $\mathrm{H}_{4} \mathrm{FBCDC}$ ligand $(500 \mathrm{mg}, 7.73 \mathrm{mmol}, 1.00$ eq.) was dissolved in $200 \mathrm{ml}$ of $\mathrm{N}, \mathrm{N}-$ Dimethylformamide (DMF) under inert atmosphere and $20 \mathrm{ml}$ of acetic acid was added. Sometimes, a part of the ligand is precipitating again after addition of the acetic acid. Careful heating of the mixture up to $80{ }^{\circ} \mathrm{C}$ supports the solution procedure in this case. Subsequently, $\mathrm{Cu}\left(\mathrm{NO}_{3}\right)_{2}\left(\mathrm{H}_{2} \mathrm{O}\right)_{2.5}(423 \mathrm{mg}, 1.82 \mathrm{mmol}, 2.50$ eq.) was added and the reaction mixture stirred for 72 $\mathrm{h}$ at $80{ }^{\circ} \mathrm{C}$ while fine green powder precipitated. After the reaction is finished the obtained powder was washed five times with DMF. A small amount of the washed precipitate was used for PXRD measurements, referred to as "as made" MOF. Desolvation of DUT-140(Cu) was achieved using a supercritical point dryer and resulted in $212 \mathrm{mg}$ (36\%) of pure and highly crystalline MOF powder.

DUT-140(Co) was synthesized using the same procedure as for the copper analogue. $\mathrm{H}_{4} \mathrm{FBCDC}$ linker $(200 \mathrm{mg}, 0.29 \mathrm{mmol}, 1.00$ eq.) was dissolved in $75 \mathrm{ml} \mathrm{N}$-Methyl-2-pyrrolidone (NMP). To this a solution cobalt nitrate hexahydrate $(212$ $\mathrm{mg}(0.73 \mathrm{mmol}, 2.50$ eq.) was added and the resulting mixture was stirred for $72 \mathrm{~h}$ at $80{ }^{\circ} \mathrm{C}$ under inert atmosphere until a brownish precipitate was formed. After completion of the reaction the crystals were washed 3 times with pure NMP. A part of the product was separated for "as made" PXRD. No further investigation of DUT140(Co) was conducted since it was solely prepared to obtain DUT-140(Zn).

DUT-140(Zn) was produced by post-synthetic metal exchange from DUT-140(Co). Therefore, the solvent above the washed DUT-140(Co) crystals 
was exchanged by a $0.1 \quad M$ solution of $\mathrm{Zn}\left(\mathrm{NO}_{3}\right)_{2}\left(\mathrm{H}_{2} \mathrm{O}\right)_{6}$. This solution was exchanged six times over two days. During the first three exchange steps, the supernatant solution turned violet. After the color completely faded, the zinc nitrate solution was refreshed three more times and the product subsequently washed three times with NMP.

\section{In situ fluorescence measurements upon desolvation}

The in situ desolvation experiment was performed in a solid-state sample holder filled with solvated material in acetone. The sample holder was positioned in $45^{\circ}$ between the incident beam and the detector. The solvent was evaporated over $25 \mathrm{~h}$ and fluorescence spectra were measured every $5 \mathrm{~min}$.

\section{In situ PXRD upon desolvation}

The powder of DUT-140(Zn) in ethanol was prepared using a background-free holder for measurements under inert conditions and covered by $7.5 \mu \mathrm{m}$ thick Kapton $\AA$ foil to ensure a slow solvent evaporation. The measurements were performed on Empyrean 2 diffractometer (Panalytical), using $\mathrm{Cu}-\mathrm{K} \alpha 1$ irradiation and Pixcel3D detector. 173 measurements were performed in the $2 \theta$ range of $7-10^{\circ}$ with exposition time of $100 \mathrm{~s}$ for each measurement.

\section{RESULTS AND DISCUSSION}

\section{In silico investigations of DUT-140}

The contraction/reopening mechanism responsible for NGA in DUT-49 is related to a deformation of the BBCDC linker, further denoted as ligand buckling. ${ }^{22}$ In previous work, a method was developed to allow an investigation of the mechanical properties of ligands, which directly reflect the switchability and the probability of NGA in frameworks isoreticular to DUT-49. ${ }^{20,24}$ This was achieved by calculating stress-strain curves for the single ligand using density functional theory (DFT) methods. Therefore, simulations were performed also for $\mathrm{H}_{4} \mathrm{FBCDC}$ ligand before synthetic efforts were made.

The mechanical properties of the $\mathrm{H}_{4} \mathrm{FBCDC}$ were determined by simulated compression of the linker at the carbazole nitrogen's (orange arrows, Figure 1a, b) using the accurate tight-binding quantum chemical method, GFN2_xtb. ${ }^{40}$ From these calculations stress applied to the linker can be determined and the mechanical stability in addition to the response of network induced pressure can be explored as shown for $\mathrm{H}_{4} \mathrm{BBCDC}$ (blue) and $\mathrm{H}_{4} \mathrm{FBCDC}$ (orange) in Figure 1c. Initially increasing the compressive strain to both linkers results in an elastic response up to a certain point, defining the maximal stress. After reaching this point, the linker itself starts to buckle (Figure 2 ). This step in the stress strain curve is a common feature of all ligands, used for the synthesis of NGA materials. ${ }^{24}$ As derived from the graph in Figure 1c the maximum stress for each linker is reached at approximately 0.014 compressive strain. The $\mathrm{H}_{4} \mathrm{FBCDC}$ ligand reaches the maximum yield stress at $1.30 \mathrm{nN}$, which is higher compared to the $\mathrm{H}_{4} \mathrm{BBCDC}$ that demonstrates an inelastic transition at $1.02 \mathrm{nN}$. Furthermore, the Young's modulus, reflected by the linear slope of the stress-strain curve, is also larger for $\mathrm{H}_{4} \mathrm{FBCDC}$. The demonstrated strain resistance behavior shows that the intended linker is more rigid than the DUT-49 linker, which follows chemical intuition based on comparison of the chemical structures. 
a

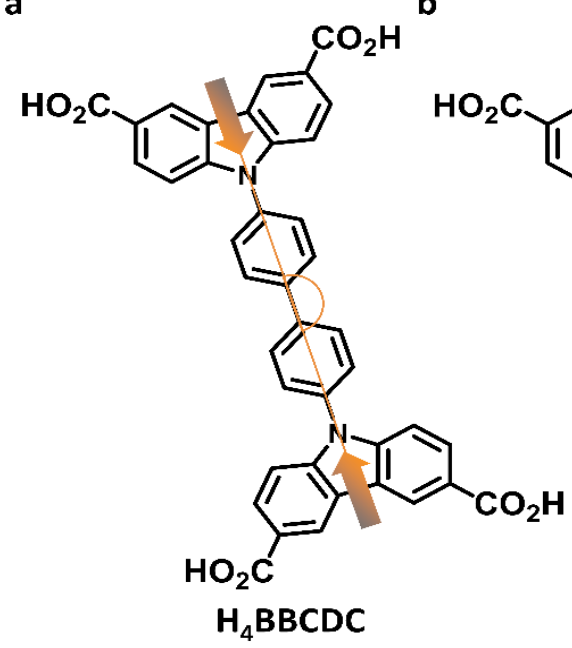

C

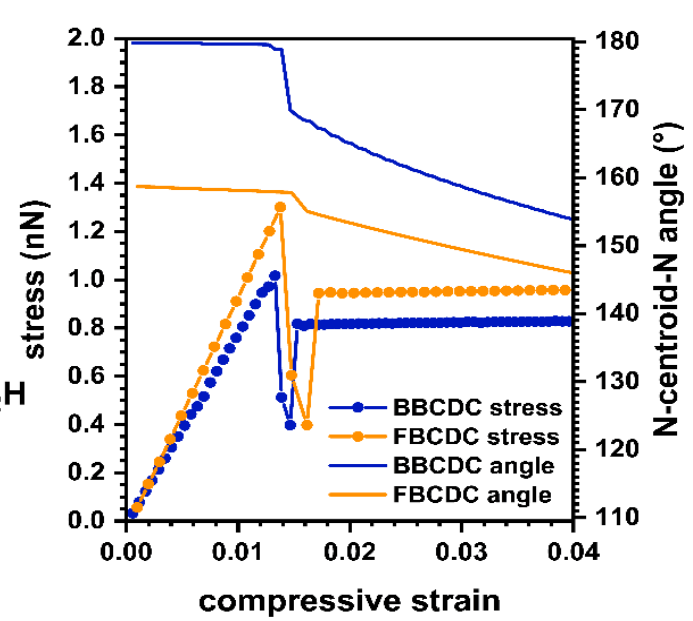

Figure 1. $\mathrm{H}_{4} \mathrm{BBCDC}(\mathrm{a})$ and $\mathrm{H}_{4} \mathrm{FBCDC}$ linker molecules (b). (c) Stress-strain curve of the ligands (dotted line). Direction of the applied pressure is indicated by the orange arrows. Evolution of $\mathrm{N}$-centroid-N angle of the ligands (solid line) while strain is applied.

These simulations also demonstrate the overall deflection for the $\mathrm{H}_{4} \mathrm{BBCDC}$ is larger than for $\mathrm{H}_{4} \mathrm{FBCDC}$. The pore sizes of the related op-phases of corresponding MOFs do not differ significantly, as shown in the supplementary information section 3.3.7. The performance of an NGA material cannot be directly derived from the stress strain curve, however, the increased rigidity of $\mathrm{H}_{4} \mathrm{FBCDC}$ is expected to permit a overloading of the metastable state, increasing the NGA step. However, increased rigidity may also lead to a reduction of the ligand's deflection which in turn could lead to a hindered contraction.

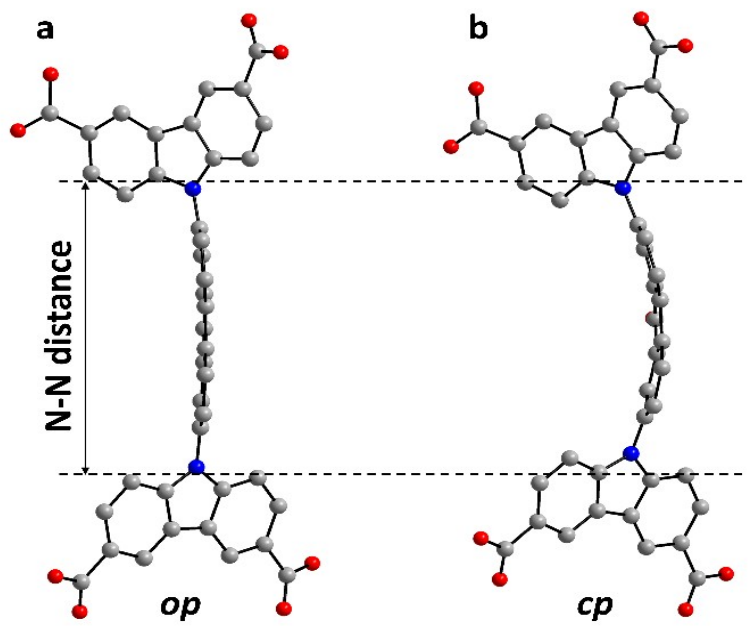

Figure 2. Deflection of $\mathrm{H}_{4} \mathrm{FBCDC}$ ligand at $0.00 \mathrm{nN}(\mathrm{a})$ and $0.03 \mathrm{nN}$ (b) compressive strain. Colour code: grey: carbon, blue: nitrogen, red: oxygen. Hydrogen atoms are omitted for clarity. 

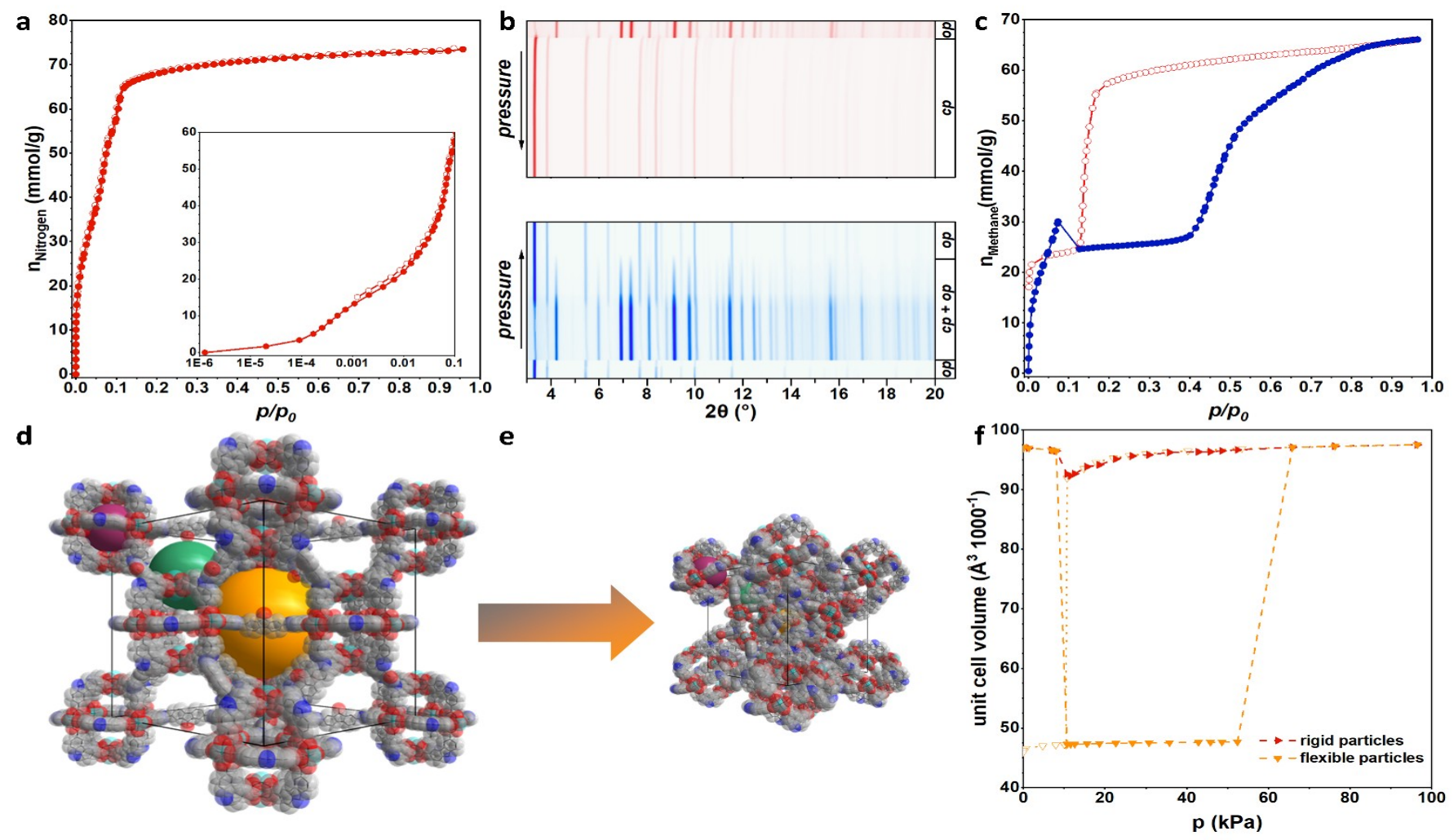

Figure 3. Crystal structure and adsorption-induced switching in DUT-140(Cu). (a) Physisorption of nitrogen on DUT-140(Cu) at $77 \mathrm{~K}$ (inset shows the low-pressure range in semilogarithmic scale); (b, c) In situ PXRD patterns and corresponding physisorption isotherm measured upon adsorption (blue) and desorption (red) of methane at $111 \mathrm{~K}$; (d) Crystal structure of the op phase of DUT-140(Cu); (e) Crystal structure of the $\mathrm{cp}$ phase of DUT-140(Cu). Voids are shown as colored spheres (purple: cuboctahedral, green: tetrahedral, and orange: octahedral cavities); (f) Evolution of the unit cell volume upon physisorption of methane on DUT-140(Cu) at $111 \mathrm{~K}$.

\section{Experimental investigation of desolvated DUT-140(Cu)}

As the theoretical calculations of the mechanical properties of $\mathrm{H}_{4} \mathrm{FBCDC}$ predict the potential for NGA, the ligand was synthesized in a similar six step approach used for the synthesis of recently reported elongated versions of $\mathrm{H}_{4} \mathrm{BBCDC}^{24}$ The carbazole was converted to dibutyl-9H-carbazole-dicarboxylate over four steps and the obtained dicarboxylate was used as starting material in an Ullman-coupling reaction with 2,7-dibromofluorenone. After the following hydrolysis and purification, the $\mathrm{H}_{4} \mathrm{FBCDC}$ ligand was obtained in $28 \%$ yield over six steps. All compounds have been analyzed by ${ }^{1} \mathrm{H}$ - and ${ }^{13} \mathrm{C}$ NMR as well as by mass spectroscopy and IRspectroscopy as shown in supplementary information chapter 2.

Combination of $\mathrm{H}_{4} \mathrm{FBCDC}$ with copper(II)nitrate in $\mathrm{N}, \mathrm{N}$-Dimethylformamide (DMF) yields the framework with a composition [ $\left.\mathrm{Cu}_{2}(\mathrm{FBCDC})\right]$ $\left(\mathrm{H}_{2} \mathrm{O}\right)_{x}(\mathrm{DMF})_{y}$, denoted as DUT-140(Cu). The MOF was synthesized using two different synthesis routes yielding crystalline powders with an average particle size of $14 \mu \mathrm{m}$ and single crystal sample with crystals up to $100 \mu \mathrm{m}$ (supporting information chapters 3.1 and 3.3.5). The crystal structure of the DUT-140(Cu) was determined by means of synchrotron single crystal X-ray diffraction at MX BL14.3 beamline of the Bessy II light source. DUT-140(Cu) crystallizes in cubic space group Fm3́m (No 225) with lattice parameter $a=46.14 \AA$ and is isostructural to DUT-49. Detailed analysis of the ligand molecule in the crystal structure shows elongated thermal ellipsoids even for carbazole moiety, indicating disorder that can be caused by vibration of the molecule in the direction perpendicular to the carbazole plane. Since the symmetry of the fluorenone backbone is lower than the symmetry of the corresponding position in the space group, the fluorenone moiety is disordered over four positions.

The material has a hierarchical pore system with three different voids (11 $\AA, 16 \AA, 24 \AA)$. The phase purity of DUT-140(Cu) was confirmed by PXRD (supporting information, chapter 3.3). The activated powder sample of DUT 140(Cu), was 
subjected to physisorption experiments using nitrogen at $77 \mathrm{~K}$ and methane at $111 \mathrm{~K}$ as probe molecules (Figure 3a, c). The low-pressure range of nitrogen isotherm, shown in Figure $3 a$, displays superimposing steps up to $p / p_{0}=0.1$ indicating the consecutive filling of the pores before reaching saturation. The desorption branch shows no hysteresis and therefore no indications of the structural flexibility. The isotherm reaches saturation at $73.5 \mathrm{mmol} / \mathrm{g}$, which is slightly lower than theoretical uptake of $74.6 \mathrm{mmol} / \mathrm{g}$. The shape of methane adsorption isotherm at $111 \mathrm{~K}$ (Figure 3c) is similar to the methane isotherm for DUT-49 at the same temperature. In the lowpressure range, the stepwise pore filling occurs up to $p / p_{0}=0.075$, at which NGA event is observed with $\Delta \mathrm{n}_{\mathrm{NGA}}=5.45 \mathrm{mmol} / \mathrm{g}$. The second step is observed at relative pressures between 0.4 and 0.6 and is associated with structure reopening in the case of DUT-49. The isotherm reaches the second plateau at $p / p_{0}=0.8$ with a resulting adsorption capacity of $63.4 \mathrm{mmol} / \mathrm{g}$. The desorption branch of the isotherm is characterized by a plateau at $p / p_{0}=0.9-0.1$, which is followed by the steep desorption step and the second plateau at $p / p_{0}<0.1$. The intersection range of adsorption and desorption branches indicates the metastability range for the structure (Figure 3c). To monitor the structural transitions upon adsorption of methane at $111 \mathrm{~K}$, the same experiment was reproduced in the external adsorption cell, inserted in the synchrotron beam of KMC-2 beamline (BESSY II). The PXRD patterns collected are shown as profile contour plots in Figure 3b. PXRD patterns confirm structural contraction from op to $\mathrm{cp}$ phase upon NGA event, showing shift of 111 reflection towards higher $2 \theta$ angle, similar as it was observed for DUT-49. In the relative pressure range of $0.1-0.5$, a mixture of op and $\mathrm{cp}$ phase is observed that can be explained by broad crystallite size distribution, a phenomenon known for DUT-49. ${ }^{23}$

Detailed analysis of the crystal size distribution (supplementary information, chapter 3.3.5) shows a distinct number of crystallites smaller than 1 $\mu \mathrm{m}$, which was defined as a critical particle size for structural transition in DUT-49 during adsorption of nitrogen at $77 \mathrm{~K}$. However, since DUT-140(Cu) shows higher resistance to the adsorption stress, the particle size dependency could be transferred to the adsorption of methane at $111 \mathrm{~K}$. The second step in the adsorption branch is characterized by the structural reopening. Interestingly, during the desorption no intermediated phases (ip) were observed, as it is known for DUT-49. ${ }^{20}$ After complete desorption of methane at $111 \mathrm{~K}$, DUT-140 stays in the cp phase. The monitoring of the cell volume also points out that even the rigid part of the sample, namely the smaller crystals, shows a slight response to the stress associated with the adsorption of methane (Figure 3f).

\section{Fluorescence in DUT-140(Cu)}

Photoluminescent properties of the $\mathrm{H}_{4} \mathrm{FBCDC}$ linker in solution and within the suspended metalorganic framework in the solid state were investigated at room temperature. According to the fluorescence spectra (Figure 4b), a $3 \mathrm{mM}$ solution of $\mathrm{H}_{4} \mathrm{FBCDC}$ linker in NMP shows a yellow emission with maximum at $17,750 \mathrm{~cm}^{-1}\left(\lambda_{\text {Ext. }}=\right.$ $\left.27,397 \mathrm{~cm}^{-1}\right)$. However, no fluorescence of the solid linker powder can be observed. This fluorescence quenching in the solid state is most likely due to the efficient $\pi$ - $\pi$-stacking of the linkers. In contrast, in the crystal of DUT-140 the MOPs are interconnected by the fluorenone containing backbones into a three-dimensional structure. As a result, the fluorenone moieties are isolated from other groups and therefore not involved in any weak interactions, which prevents a

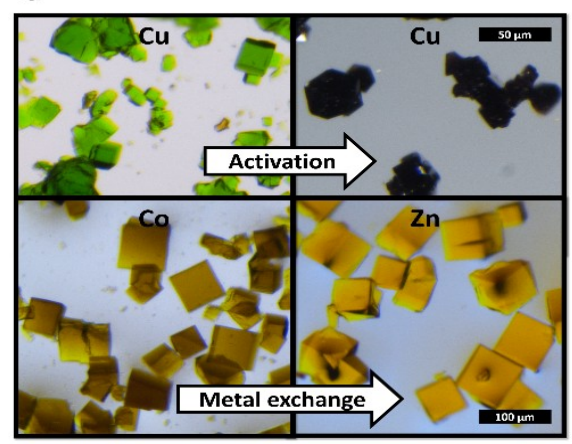

b

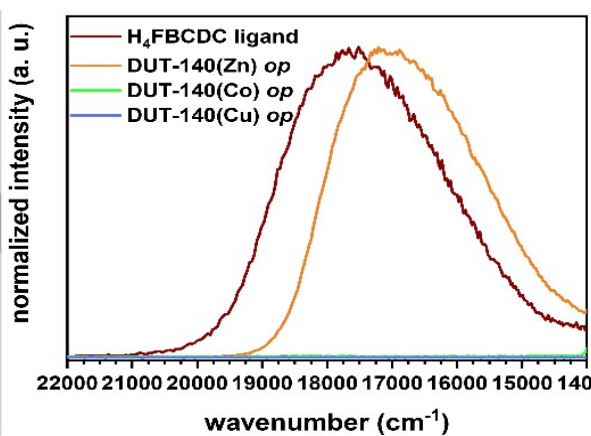

c

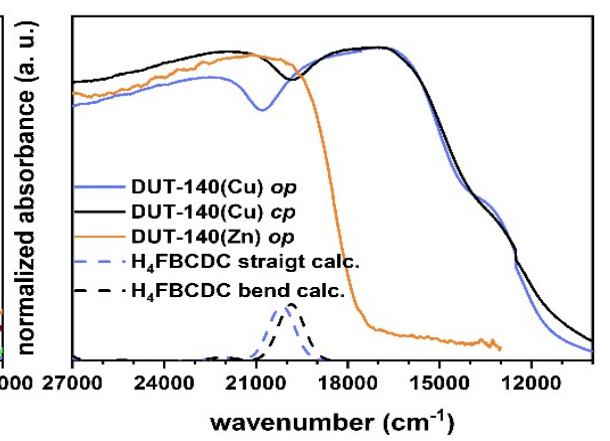

Figure 4. (a) Microscopy images of DUT-140(M) crystals, top line: DUT-140(Cu) before and after activation, bottom line DUT-140(Co) after synthesis and DUT140(Zn) after metal exchange. (b) Fluorescence spectra of dissolved fluorenone linker (3 mM) and DUT-140(M)_op, suspended in NMP. Excitation wavelength: 365 $\mathrm{nm}$. (c) Absorption spectra of DUT-140(Cu) op- and cp-phases and DUT-140(Zn)_op. Dotted lines correspond to a specific excitation in the stretched (blue) and contracted (black) form of the $\mathrm{H}_{4} \mathrm{FBCDC}$, respectively. 
fluorescence quenching as it is shown in figure S11 of the supplementary information. However, $d-d$ transitions in the $d^{9} \mathrm{Cu}^{2+}$ ions still hinder a spectroscopic investigation of the linker in the network. As a result, no fluorescence can be detected after crystallization of the MOF, as this is suppressed due to the complex formation and quenching.

Nevertheless, the UV/Vis spectra of the DUT$140(\mathrm{Cu})$ in the op and $\mathrm{cp}$ phases were recorded and compared with simulated UV/Vis spectra of the straight and bent ligand (Figure 4c). DUT-140(Cu) shows a strong absorption in the range of $15,000 \mathrm{~cm}^{-1}$ to $27,500 \mathrm{~cm}^{-1}$. This absorption is divided by a small local minimum whereby the absorption band at higher wavenumbers can be attributed to the linkerabsorption and the absorption band at lower wavenumbers originates from the metal centers.

The contraction of the DUT-140(Cu) from the op (blue line) to the $\mathrm{cp}$ (black line) phase causes a red shift of the first absorption maximum. The simulated absorption spectra of the linker in the stretched and bent forms (Fig. 4c dotted lines) shows the same trend for the shift in absorbance for a $\pi-\pi^{*}$ excitation from the carbazoles to the fluorenone system (Fig. S12) at around $20,000 \mathrm{~cm}^{-1}$. This observation indicates changes in the electronic structure of the linker during contraction and an excitation at lower energies for the $\mathrm{cp}$ phase of the network.

\section{Metal exchange}

In order to detect the fluorescence of the metalorganic framework, it is necessary to replace copper by a spectroscopically "silent" metal, which is similarly capable of forming paddle wheel units. Regarding these requirements $\mathrm{Zn}^{2+}$ is a suitable target metal because of $\mathrm{d}^{10}$ electronic configuration. However, a direct synthesis of DUT-140(Zn) starting from the linker and zinc salt was not successful.

Therefore, the metal exchange approach, recently reported by our group for DUT- $49,{ }^{41}$ was used and first attempts were made to synthesize the cobalt-based DUT-140 using NMP as a solvent. The obtained single crystals were repeatedly washed with fresh NMP and afterwards the brownish DUT-140(Co) crystals were exposed to $0.1 \mathrm{M}$ zinc nitrate solution in NMP. The change of the crystal color from initial brown to a distinct orange with simultaneous violet coloration of the supernatant solution indicates the metal exchange. To confirm the complete metal exchange EDX measurements were conducted as shown in supplementary figure 7. Figure 4a shows the color evolution of the crystals during the metal exchange procedure. Furthermore, it can be clearly seen that the cubic morphology of the crystals remains after the metal exchange. Single crystal X-ray diffraction experiments reveals that DUT-140(Co) and DUT-140(Zn) are isostructural to DUT $140(\mathrm{Cu})$. The crystal structure of both materials was solved in space group Fm3́m (No225) with lattice parameters $a=46.47 \AA$ for DUT-140(Co) and $a=46.52 \AA$ for DUT $140(\mathrm{Zn})$. The crystal structures confirm the trend for the $M$ $M$ distance within the paddle wheel, previously observed in the DUT-49(M) frameworks and dictated by Irving-Williams series showing increasing $M-M$ distance in the series DUT140 (Cu) < DUT-140(Co) < DUT-140(Zn). ${ }^{41}$

In addition to the synthesis of a new responsive MOF and the successful metal replacement, the initial goal was finally achieved by this procedure as shown in Figure $4 \mathrm{~b}$. In addition to DUT-140(Cu) (blue line) and DUT-140(Co) (green line), which are both non-luminescent, the fluorescent properties of the linker in the DUT-140(Zn) (orange line) were observed.

\section{Fluorescence in DUT-140(Zn)}

In comparison to the ligand, the spectrum of DUT-140(Zn) shows a red shifted emission maximum at $17,000 \mathrm{~cm}^{-1}$. This behavior is attributed to coordination to the metal center. Since suspended crystals were used to record fluorescence spectra a comparison of luminescence intensity with that of the dissolved linker is not feasible.

Unfortunately, the desolvation of the DUT140 (Zn) framework even using supercritical $\mathrm{CO}_{2}$ drying leads to amorphization of the material. Therefore, studies on the guest-free DUT-140(Zn) are not possible at this stage and the following experiments were performed on solvated crystals.

\section{Time resolved desolvation experiment}

Garai et al. demonstrated a structural transition from op to intermediate (ip) phase taking place upon solvent desorption in case of DUT-49(Zn), identified by time resolved in situ PXRD. ${ }^{42}$ Therefore we conducted two experiments to investigate the effect of desolvation on the

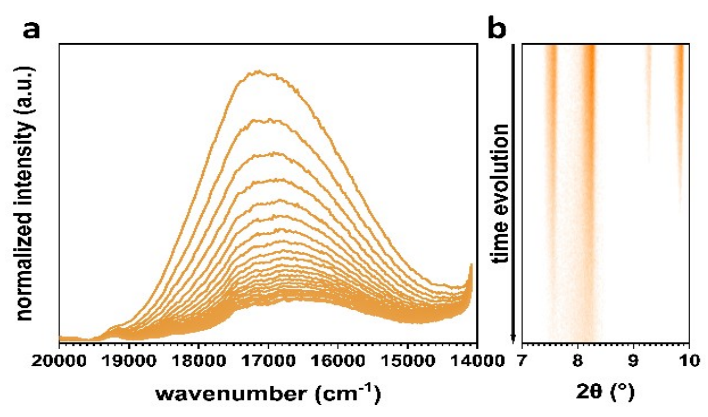

Figure 5. Time dependent desolvation experiments on DUT140(Zn). (a) fluorescence decay over desolvation from Acetone. Spectra recorded every $5 \mathrm{~min}$ for 25 h. (b) in situ PXRD over desolvation from EtOH. PXRD was recorded every 115 s over $5.5 \mathrm{~h}$. 
fluorescence properties of DUT-140(Zn). At first, the solvent in the pores of as made DUT-140(Zn) was replaced by acetone. In an in situ experiment, the evaporation of acetone was followed by fluorescence spectroscopy.

Figure $5 \mathrm{a}$ shows the fluorescence decay during desolvation from acetone. The spectra show two main features: a decrease in fluorescence intensity during desolvation and second, a slight red shift of the fluorescence maximum from about $17,000 \mathrm{~cm}^{-1}$ to about $16,500 \mathrm{~cm}^{-1}$. Accordingly, there is a change in the fluorescence properties during the desolvation of the material. To examine whether this change is related to a structural change of the material, an in situ PXRD experiment was performed. The contour plot in Figure $5 b$ does not show a shift of the individual reflections with time. Therefore, no structural contraction to another crystalline phase can be assumed for DUT-140(Zn) compared to DUT-49(Zn), showing transition from op to ip phase upon solvent desorption before amorphization. ${ }^{42}$

Although all previous experiments clearly indicate that there might be a change in fluorescence due to the structural change of the material, no evidence for this hypothesis can be found from these experiments and the redshift can be attributed to the amorphization and the rupture of some coordination bonds.

This behavior is most likely caused by the fine interplay of the lower stability of the zinc paddle wheel unit compared to the copper paddle wheel unit and the higher rigidity of the $\mathrm{H}_{4} \mathrm{FBCDC}$ linker (DUT-140) compared to the $\mathrm{H}_{4} \mathrm{BBCDC}$ linker (DUT49). The more rigid $\mathrm{H}_{4} \mathrm{FBCDC}$ linker does not even allow a small contraction of the network due to desorption stress and capillary forces before the coordination bond within the zinc paddlewheel breaks. For DUT-49(Zn) in comparison, the difference between the paddlewheel stability and the linker rigidity is exactly this much smaller a contraction can happen before the coordination bond breaks.

This finding leads to the assumption that a linker with smaller rigidity than that of DUT-49 linker could lead to a network that shows a full contraction before amorphization while solvent evaporation.

\section{CONCLUSION}

In brief, three new MOF materials DUT-140(M) $(M=\mathrm{Cu}, \mathrm{Co}, \mathrm{Zn})$ were successfully synthesized. As predicted by mechanical considerations of the $\mathrm{H}_{4} \mathrm{FBCDC}$ ligand, the metal-organic framework DUT-140(Cu) shows intrinsic flexibility as well as negative gas adsorption property with an $\Delta n_{\text {NGA }}=5.45 \mathrm{mmol} / \mathrm{g}$. The contraction of the opphase can be induced by adsorption of hydrocarbons like methane or butane as well as carbon dioxide. Adsorption experiments also confirm the assumption that the use of the
$\mathrm{H}_{4} \mathrm{FBCDC}$ linker leads to a more rigid network since the adsorption of nitrogen does not trigger the phase transformation.

UV/Vis investigations of DUT-140(Cu) showed a distinct change in the absorption spectrum which could be attributed to an excitation from the carbazole moiety to the fluorenone part by DFT calculations. Since this excitation is highly affected by the deformation of the linker, it can be concluded that the fluorescence spectrum also should change under the given conditions due to the structural transformation.

The fluorescence properties of the fluorescent linker could be unveiled by an exchange of the metal node to a zinc paddlewheel. However, the stiffening of the metal-organic framework did not lead to the desired increased resistance to desolvation stress for DUT-140(Zn), indicating the metal node to be the main limiting factor, leading to amorphization during desolvation in such highly porous framework materials, even under mild supercritical conditions.

In situ fluorescence studies provide clear evidence that a fluorescence change is caused by structural decomposition of the metal-organic framework and revealed the importance of the fine interplay of linker rigidity and metal node stability for the investigation of structural contraction by solvent desorption.

Overall, fluorenone moieties are valuable functional building blocks for the detection of structural dynamics in the organic sublattice of porous framework compounds.

\section{ASSOCIATED CONTENT}

Supporting Information. Supporting information can be accessed free of charge on the ACS Publication website at DOI:

Synthesis and characterization of organic compounds, PXRD, SEM, EDX, TGA.

Crystallographic data for DUT-140(Cu), DUT-140(Zn) and DUT-140(Co) (CCDC-2080449-208045) can be obtained free of charge from the Cambridge Crystallographic Data Centre via www.ccdc.cam.ac.uk/data_request/cif. Adsorption information files (.aif) for nitrogen (77K) and methane $(111 \mathrm{~K})$ are enclosed as supporting data.

\section{ABBREVIATIONS}

DUT, Dresden University of Technology; MOF, metalorganic framework; MOP, metal-organic polyhedra; NGA, Negative Gas Adsorption; op, open pore; $c p$, contracted pore, ip, intermediate pore; $\mathrm{H}_{4} \mathrm{BBCDC}$, 9,9'-([1,1'-biphenyl]-4,4'-diyl)-bis(9H-carbazole 3,6dicarboxylic acid; $\mathrm{H}_{4} \mathrm{FBCDC}$, 9,9'-(9-oxo-9H-fluorene2,7-diyl)-bis(9H-carbazole-3,6-dicarboxylic acid; MLCT, metal-to-ligand charge transfer; LMCT, ligandto-metal charge transfer; DMF, $\mathrm{N}, \mathrm{N}$ Dimethylformamide; NMP, 1-Methylpyrrolidin-2-one; GCMC, Grand Canonical Monte Carlo;

\section{AUTHOR INFORMATION}




\section{Corresponding Author}

* Prof. Dr. Stefan Kaskel - Anorganische Chemie I, Fachrichtung Chemie und Lebensmittelchemie, Technische Universität Dresden, Bergstrasse 66, 01062 Dresden, Germany

\section{Author Contributions}

F. Walenszus synthesized, activated, and performed characterization of organic ligands and MOF samples. F. Walenszus, V. Bon, D. Wallacher, and D. $M$. Többens contributed to in situ PXRD measurements. V. Bon performed refinement of PXRD data. F. Walenszus, V. Bon, J. Evans, I. Senkovska and S. Kaskel contributed to analysis, interpretation and discussion of adsorption and single crystal X-ray diffraction data. J. D. Evans performed computational analysis of mechanical and adsorption properties. F. Schwotzer performed SEM analysis. F. Walenszus, V. Bon, J. D. Evans, I. Senkovska and S. Kaskel organized the project. All authors contributed to writing and improving the manuscript.

\section{Funding Sources}

This project has received funding from the European Research Council (ERC) under the European Union's Horizon 2020 research and innovation program (grant agreement No. 742743), the BMBF (No. 05K19OD2) and ANR/DFG (Project Number 391704421).

\section{ACKNOWLEDGMENT}

The authors thank the "Helmholtz-Zentrum Berlin für Materialien und Energie" for the allocated beam time at KMC-2 (in situ PXRD) and D. Wallacher, D. M. Többens and N. Grimm for support with the measurement setup, MX BL14.3 (single crystal X-ray diffraction) beamlines of BESSY-II and travel funding. The authors thanks Philipp Lange for performing elemental analysis. J. D. E. acknowledges the support of the Alexander von Humboldt foundation and the Center for Information Services and HighPerformance Computing $(\mathrm{ZIH})$ at TU Dresden for providing high-performance computing facilities.

\section{REFERENCES}

(1) Batten, S. R.; Champness, N. R.; Chen, X.-M.; Garcia-Martinez, J.; Kitagawa, S.; Öhrström, L.; O'Keeffe, M.; Paik Suh, M.; Reedijk, J. Terminology of metalorganic frameworks and coordination polymers (IUPAC Recommendations 2013). Pure Appl. Chem. 2013, 85 (8), 1715-1724. DOI: 10.1351/PAC-REC-12-11-20.

(2) Horike, S.; Shimomura, S.; Kitagawa, S. Soft porous crystals. Nat. Chem. 2009, 1 (9), 695-704. DOI: 10.1038/nchem.444.

(3) Furukawa, H.; Cordova, K. E.; O'Keeffe, M.; Yaghi, O. M. The Chemistry and Applications of Metal-Organic Frameworks. Science 2013, 341 (6149), 1230444. DOI: $10.1126 /$ science.1230444.

(4) Zhou, H.-C.; Long, J. R.; Yaghi, O. M. Introduction to Metal-Organic Frameworks. Chem. Rev. 2012, 112 (2), 673-674. DOI: 10.1021/cr300014x.

(5) Morris, R. E.; Wheatley, P. S. Gas Storage in Nanoporous Materials. Angew. Chem. Int. Ed. 2008, 47 (27), 4966-4981. DOI: 10.1002/anie.200703934.

(6) Bon, V.; Brunner, E.; Pöppl, A.; Kaskel, S. Unraveling Structure and Dynamics in Porous
Frameworks via Advanced In Situ Characterization Techniques. Adv. Funct. Mater. 2020, 1907847. DOI: 10.1002/adfm.201907847.

(7) Chen, Z.; Li, P.; Anderson, R.; Wang, X.; Zhang, X.; Robison, L.; Redfern, L. R.; Moribe, S.; Islamoglu, T.; Gómez-Gualdrón, D. A.; Yildirim, T.; Stoddart, J. F.; Farha, O. K. Balancing volumetric and gravimetric uptake in highly porous materials for clean energy. Science 2020, 368 (6488), 297-303. DOI: 10.1126/science.aaz8881.

(8) Kitagawa, S.; Kondo, M. Functional Micropore Chemistry of Crystalline Metal Complex-Assembled Compounds. Bull. Chem. Soc. Jpn. 1998, 71 (8), 17391753. DOI: $10.1246 /$ bcsj.71.1739.

(9) Liu, X.; Wang, M.; Zhou, S.; Wang, J.; Xin, H.; Wei, S.; Liu, S.; Wang, Z.; Lu, X. Tracking CO2 capture and separation over $\mathrm{N} 2$ in a flexible metal-organic framework: insights from GCMC and DFT simulations. J. Mater. Sci. 2021, 56 (17), 10414-10423. DOI: 10.1007/ s10853-021-05970-7.

(10) Sin, M.; Kavoosi, N.; Rauche, M.; Pallmann, J.; Paasch, S.; Senkovska, I.; Kaskel, S.; Brunner, E. In Situ 13C NMR Spectroscopy Study of $\mathrm{CO} 2 / \mathrm{CH} 4$ Mixture Adsorption by Metal-Organic Frameworks: Does Flexibility Influence Selectivity? Langmuir 2019, 35 (8), 3162-3170. DOI: 10.1021/acs.langmuir.8b03554. Published Online: Feb. 12, 2019.

(11) Dong, Q.; Zhang, X.; Liu, S.; Lin, R.-B.; Guo, Y.; Ma, Y.; Yonezu, A.; Krishna, R.; Liu, G.; Duan, J.; Matsuda, R.; Jin, W.; Chen, B. Tuning Gate-Opening of a Flexible Metal-Organic Framework for Ternary Gas Sieving Separation. Angew. Chem. Int. Ed. 2020, 59 (50), 22756-22762. DOI: 10.1002/anie.202011802. Published Online: Oct. 7, 2020.

(12) Freund, P.; Mielewczyk, L.; Rauche, M.; Senkovska, I.; Ehrling, S.; Brunner, E.; Kaskel, S. MIL53(Al)/Carbon Films for CO 2 -Sensing at High Pressure. ACS Sustainable Chem. Eng. 2019, 7 (4), 4012-4018. DOI: 10.1021/acssuschemeng.8b05368.

(13) Ingle, N.; Sayyad, P.; Deshmukh, M.; Bodkhe, G.; Mahadik, M.; Al-Gahouari, T.; Shirsat, S.; Shirsat, M. D. A chemiresistive gas sensor for sensitive detection of $\mathrm{SO} 2$ employing Ni-MOF modified -OH-SWNTs and -OHMWNTs. Appl. Phys. A 2021, 127 (2). DOI: 10.1007/s00339-021-04288-0.

(14) Yang, Y.; Li, L.; Yang, H.; Sun, L. Five LanthanideBased Metal-Organic Frameworks Built from a $\pi-$ Conjugated Ligand with Isophthalate Units Featuring Sensitive Fluorescent Sensing for DMF and Acetone Molecules. Cryst. Growth Des. 2021. DOI: 10.1021/acs.cgd.1c00116.

(15) Souza, B. E.; Donà, L.; Titov, K.; Bruzzese, P.; Zeng, Z.; Zhang, Y.; Babal, A. S.; Möslein, A. F.; Frogley, M. D.; Wolna, M.; Cinque, G.; Civalleri, B.; Tan, J.-C. Elucidating the Drug Release from Metal-Organic Framework Nanocomposites via In Situ Synchrotron Microspectroscopy and Theoretical Modeling. ACS Appl. Mater. Interfaces 2020, 12 (4), 5147-5156. DOI: 10.1021/acsami.9b21321. Published Online: Jan. 16, 2020.

(16) Suresh, K.; Matzger, A. J. Enhanced Drug Delivery by Dissolution of Amorphous Drug Encapsulated in a Water Unstable Metal-Organic Framework (MOF). Angew. Chem. Int. Ed. 2019, 58 (47), 16790-16794. DOI: 10.1002/anie.201907652. Published Online: Oct. 11, 2019.

(17) Sato, H.; Kosaka, W.; Matsuda, R.; Hori, A.; Hijikata, Y.; Belosludov, R. V.; Sakaki, S.; Takata, M.; Kitagawa, S. Self-Accelerating CO Sorption in a Soft Nanoporous Crystal. Science 2014, 343 (6167), 167170. DOI: $10.1126 /$ science. 1246423. 
(18) Aljammal, N.; Jabbour, C.; Chaemchuen, S.; Juzsakova, T.; Verpoort, F. Flexibility in Metal-Organic Frameworks: A Basic Understanding. Catalysts 2019, 9 (6), 512. DOI: 10.3390/catal9060512.

(19) Schneemann, A.; Bon, V.; Schwedler, I.; Senkovska, I.; Kaskel, S.; Fischer, R. A. Flexible metalorganic frameworks. Chem. Soc. Rev. 2014, 43 (16), 6062-6096. DOI: 10.1039/C4CS00101J.

(20) Krause, S.; Bon, V.; Senkovska, I.; Stoeck, U.; Wallacher, D.; Többens, D. M.; Zander, S.; Pillai, R. S.; Maurin, G.; Coudert, F.-X.; Kaskel, S. A pressureamplifying framework material with negative gas adsorption transitions. Nature 2016, 532 (7599), 348352. DOI: 10.1038/nature17430.

(21) Stoeck, U.; Krause, S.; Bon, V.; Senkovska, I.; Kaskel, S. A highly porous metal-organic framework, constructed from a cuboctahedral super-molecular building block, with exceptionally high methane uptake. Chem. Commun. 2012, 48 (88), 10841. DOI: 10.1039/c2cc34840c.

(22) Evans, J. D.; Bocquet, L.; Coudert, F.-X. Origins of Negative Gas Adsorption. Chem 2016, 1 (6), 873-886. DOI: 10.1016/j.chempr.2016.11.004.

(23) Krause, S.; Bon, V.; Senkovska, I.; Többens, D. M.; Wallacher, D.; Pillai, R. S.; Maurin, G.; Kaskel, S. The effect of crystallite size on pressure amplification in switchable porous solids. Nat. Commun. 2018, 9 (1), 1573. DOI: 10.1038/s41467-018-03979-2.

(24) Krause, S.; Evans, J. D.; Bon, V.; Senkovska, I.; lacomi, P.; Kolbe, F.; Ehrling, S.; Troschke, E.; Getzschmann, J.; Többens, D. M.; Franz, A.; Wallacher, D.; Yot, P. G.; Maurin, G.; Brunner, E.; Llewellyn, P. L.; Coudert, F.-X.; Kaskel, S. Towards general network architecture design criteria for negative gas adsorption transitions in ultraporous frameworks. Nat. Commun. 2019, 10 (1), 3632. DOI: 10.1038/s41467-019-11565-3.

(25) Krause, S.; Reuter, F. S.; Ehrling, S.; Bon, V.; Senkovska, I.; Kaskel, S.; Brunner, E. Impact of Defects and Crystal Size on Negative Gas Adsorption in DUT-49 Analyzed by In Situ129Xe NMR Spectroscopy. Chem. Mater. 2020, 32 (11), 4641-4650. DOI: 10.1021/acs.chemmater.0c01059. Published Online: May. 11, 2020.

(26) Kolbe, F.; Krause, S.; Bon, V.; Senkovska, I.; Kaskel, S.; Brunner, E. High-Pressure in Situ ^129 Xe NMR Spectroscopy: Insights into Switching Mechanisms of Flexible Metal-Organic Frameworks Isoreticular to DUT-49. Chem. Mater. 2019, 31 (16), 6193-6201. DOI: 10.1021/acs.chemmater.9b02003.

(27) Walenszus, F.; Bon, V.; Evans, J. D.; Kaskel, S.; Dvoyashkin, M. Molecular Diffusion in a Flexible Mesoporous Metal-Organic Framework over the Course of Structural Contraction. J. Phys. Chem. Lett. 2020, 11 (22), 9696-9701. DOI: 10.1021/acs.jpclett.0c02745. Published Online: Nov. 2, 2020.

(28) Krause, S.; Evans, J. D.; Bon, V.; Senkovska, I.; Coudert, F.-X.; Többens, D. M.; Wallacher, D.; Grimm, N.; Kaskel, S. The role of temperature and adsorbate on negative gas adsorption transitions of the mesoporous metal-organic framework DUT-49. Faraday discussions 2021, 225 (0), 168-183. DOI: 10.1039/d0fd00013b.

(29) Bon, V.; Krause, S.; Senkovska, I.; Grimm, N.; Wallacher, D.; Többens, D. M.; Kaskel, S. Massive pressure amplification by stimulated contraction of mesoporous frameworks. Angew. Chem. Int. Ed. 2021. DOI: 10.1002/anie.202100549. Published Online: Mar. 2, 2021.
(30) Schaber, J.; Krause, S.; Paasch, S.; Senkovska, I.; Bon, V.; Többens, D. M.; Wallacher, D.; Kaskel, S.; Brunner, E. In Situ Monitoring of Unique Switching Transitions in the Pressure-Amplifying Flexible Framework Material DUT-49 by High-Pressure ^129 Xe NMR Spectroscopy. J. Phys. Chem. C 2017, 121 (9), 5195-5200. DOI: 10.1021/acs.jpcc.7b01204.

(31) Allendorf, M. D.; Bauer, C. A.; Bhakta, R. K.; Houk, R. J. T. Luminescent metal-organic frameworks. Chem. Soc. Rev. 2009, 38 (5), 1330. DOl: $10.1039 / \mathrm{b} 802352 \mathrm{~m}$.

(32) Hu, Z.; Deibert, B. J.; Li, J. Luminescent metalorganic frameworks for chemical sensing and explosive detection. Chem. Soc. Rev. 2014, 43 (16), 5815-5840. DOI: 10.1039/C4CS00010B.

(33) Anik, Ü.; Timur, S.; Dursun, Z. Metal organic frameworks in electrochemical and optical sensing platforms: a review. Microchim. Acta 2019, 186 (3), 196. DOI: 10.1007/s00604-019-3321-0.

(34) Newsome, W. J.; Chakraborty, A.; Ly, R. T.; Pour, G. S.; Fairchild, D. C.; Morris, A. J.; Uribe-Romo, F. J. Jdimer emission in interwoven metal-organic frameworks. Chem. Sci. 2020, 10.1039.D0SC00876A. DOI: 10.1039/D0SC00876A.

(35) Yang, L.; Song, Y.; Wang, L. Multi-emission metalorganic framework composites for multicomponent ratiometric fluorescence sensing: recent developments and future challenges. J. Mater. Chem. Blt 2020, 10.1039.C9TB01931F. DOI: 10.1039/C9TB01931F.

(36) Wei, Z.; Chen, D.; Guo, Z.; Jia, P.; Xing, H. Eosin YEmbedded Zirconium-Based Metal-Organic Framework as a Dual-Emitting Built-In Self-Calibrating Platform for Pesticide Detection. Inorg. Chem. 2020 acs.inorgchem.9b03635. 10.1021/acs.inorgchem.9b03635.

(37) Li, X.; Yu, J.; Gosztola, D. J.; Fry, H. C.; Deria, P. Wavelength-Dependent Energy and Charge Transfer in MOF: A Step toward Artificial Porous Light-Harvesting System. J. Am. Chem. Soc. 2019, 141 (42), 1684916857. DOI: 10.1021/jacs.9b08078.

(38) Cui, Y.; Yue, Y.; Qian, G.; Chen, B. Luminescent Functional Metal-Organic Frameworks. Chem. Rev. 2012, 112 (2), 1126-1162. DOI: 10.1021/cr200101d.

(39) Sabbatini, N.; Guardigli, M.; Lehn, J.-M. Luminescent lanthanide complexes as photochemical supramolecular devices. Coord. Chem. Rev. 1993, 123 (1-2), 201-228. DOI: 10.1016/0010-8545(93)85056-A.

(40) Bannwarth, C.; Ehlert, S.; Grimme, S. GFN2-xTB -An Accurate and Broadly Parametrized Self-Consistent Tight-Binding Quantum Chemical Method with Multipole Electrostatics and Density-Dependent Dispersion Contributions. J. Chem. Theory Comput. 2019, 15 (3), 1652-1671. DOI: 10.1021/acs.jctc.8b01176.

(41) Garai, B.; Bon, V.; Krause, S.; Schwotzer, F.; Gerlach, M.; Senkovska, I.; Kaskel, S. Tunable Flexibility and Porosity of the Metal-Organic Framework DUT-49 through Postsynthetic Metal Exchange. Chem. Mater. 2020, 32 (2), 889-896. DOI: 10.1021/acs.chemmater.9b04769.

(42) Garai, B.; Bon, V.; Walenszus, F.; Khadiev, A.; Novikov, D. V.; Kaskel, S. Elucidating the Structural Evolution of a Highly Porous Responsive Metal-Organic Framework (DUT-49(M)) upon Guest Desorption by Time-Resolved in Situ Powder X-ray Diffraction. Cryst. Growth Des. 2020, acs.cgd.0c01080. DOI: 10.1021/acs.cgd.0c01080. 